\title{
Tuned MSSM Higgses as an inflaton
}

\author{
Arindam Chatterjee ${ }^{1}$ and Anupam Mazumdar ${ }^{2,3}$ \\ 1 Physikalisches Institut der Universität Bonn, Nußallee 12, Bonn, 53115, Germany \\ 2 Physics Department, Lancaster University, LA1 $4 Y B$, UK \\ 3 Niels Bohr Institute, Copenhagen University, Blegdamsvej-17, Denmark
}

\begin{abstract}
We consider the possibility that the vacuum energy density of the MSSM (Minimal Supersymmetric Standard Model) flat direction condensate involving the Higgses $H_{1}$ and $H_{2}$ is responsible for inflation. We also discuss how the finely tuned Higgs potential at high vacuum expectation values can realize cosmologically flat direction along which it can generate the observed density perturbations, and after the end of inflation - the coherent oscillations of the Higgses reheat the universe with all the observed degrees of freedom, without causing any problem for the electroweak phase transition.
\end{abstract}

\section{INTRODUCTION}

Inflationary paradigm has been extremely successful from observational point of view as it is responsible for stretching the initial perturbations to the present Hubble scales [1]. Since all relevant matter, such as the Standard Model (SM) degrees of freedom, has to be created after the end of inflation, it is obvious that the inflaton vacuum cannot be arbitrary, for a review on inflation see [2]. Inflation may occur at different scales and at different vacua, but the last phase of inflation must create all matter and observed perturbations in the cosmic microwave background (CMB) radiation [2].

In order to achieve this one has to realize inflation within a visible sector where all the couplings are well known. The MSSM (Minimal Supersymmetric Standard Model) inflation is one such example where inflation is driven by gauge invariant combinations of $D$-flat directions 3 [5]. The inflaton decays directly into the SM quarks and leptons, thus creating the baryons [6], and also the cold dark matter particles-as one of the lightest supersymmetric particle (LSP) 7, 8]. These models of inflation also solve the generic moduli problem- with a $\mathrm{TeV}$ mass moduli field is typically heavy during inflation, and thus never get displaced from their minima, or their coherent oscillations die-off during inflation [3 $\left[\begin{array}{l}5 \\ 5\end{array}\right]^{1}$.

In this paper our aim will be to show that under some fine-tuned conditions the MSSM Higgses can also drive inflation, their slow rolling can create seed perturbations for the CMB, and excite all the relevant matter for the

\footnotetext{
${ }^{1}$ Inflation may as well proceed in many hidden sectors beyond the $\mathrm{SM}$, and at sufficiently high scales. The main obstacle such models face is how to reproduce the SM baryons and the observed abundance of dark matter 2] from completely arbitrary scalar fields with couplings usually set by hand. In order to construct such hidden sector models we must know all the inflaton couplings to hidden and visible matter, and a proper embedding of inflation with a UV complete theory. One such unique model has been constructed within type IIB string theory, where it was found that all the inflaton energy is transferred to exciting the hidden matter [9], and the universe could be prematurely dominated by hidden sector dark matter.
}

success of cosmology in the visible sector. Although the Higgs potential would require fine tuning in maintaining the flatness at the scale of inflation determined by the VEV, $10^{14} \mathrm{GeV}$, but at the weak scale due to the running of the masses and couplings the Higgs can facilitate an electroweak phase transition within a large parameter space of the MSSM.

In section II, we will discuss the Higgs potential, in section III, we will discuss how inflation can be driven near the point of inflection, and describe the parameter space which leads to a successful cosmology, in section IV, we will discuss the issue of fine tuning and the electroweak symmetry breaking.

\section{THE POTENTIAL}

Let us begin by considering the $\mu$ term of the MSSM superpotential. Besides MSSM superpotential, it is also possible to have non-renormalizable terms of the following form in the superpotential [11, 12 $]^{2}$,

$$
\mathcal{W}=\mu \mathbf{H}_{\mathbf{1}} \cdot \mathbf{H}_{\mathbf{2}}+\frac{\lambda_{k}}{k} \frac{\left(\mathbf{H}_{\mathbf{1}} \cdot \mathbf{H}_{\mathbf{2}}\right)^{k}}{M_{P}^{2 k-3}},
$$

where $\mathbf{H}_{\mathbf{1}}$ and $\mathbf{H}_{\mathbf{2}}$ are Higgs superfields, $M_{P}$ denotes the reduced Planck mass, equals to $2.4 \times 10^{18} \mathrm{GeV}$. The scalar Higgs fields, $H_{1}, H_{2}$, which give masses to the down-type and up-type quarks respectively, constitutes the scalar components of $\mathbf{H}_{\mathbf{1}}$ and $\mathbf{H}_{\mathbf{2}}$ respectively. In the above equation $k \geq 2$. For simplicity, we begin by considering one such non-renormalizable term at a time.

We then obtain the scalar potential along the $H_{1} H_{2}$ D-flat direction,

$$
\begin{aligned}
\tilde{V}(\varphi, \theta) & =\frac{1}{2} m^{2}(\theta) \varphi^{2}+(-1)^{(k-1)} 2 \lambda_{k}^{\prime} \mu \cos ((2 k-2) \theta) \varphi^{2 k} \\
& +2 \lambda_{k}^{\prime 2} \varphi^{2(2 k-1)}
\end{aligned}
$$

\footnotetext{
2 The $\mu$-term has been considered in past to generate the density perturbations, see [13].
} 
where $\phi=|\phi| e^{i \theta}, \varphi=\sqrt{2}|\phi|$, and

$$
\begin{aligned}
H_{1} & =\frac{1}{\sqrt{2}}(\phi, 0)^{T}, \\
H_{2} & =\frac{1}{\sqrt{2}}(0, \phi)^{T}, \\
m^{2}(\theta) & =\frac{1}{2}\left(m_{1}^{2}+m_{2}^{2}+2 \mu^{2}-2 B \mu \cos 2 \theta\right), \\
\lambda_{k}^{\prime} & =\frac{\lambda_{k}}{2^{(2 k-1)} M_{P}^{2 k-3}} .
\end{aligned}
$$

For simplicity here we assume $\mu$ and $B$ to be real. This choice is compatible with the experimental constraints, mainly from the EDM measurements and is well motivated, especially in the context of weak scale supersymmetry [10].

The potential is bounded from below for $m^{2} \geq \mu^{2}$, and the minimum is 0 at $\varphi=0$. However, it is also possible to have local extrema. For any $\lambda_{k}$, we have

$$
\begin{aligned}
\frac{\partial \tilde{V}}{\partial \theta} & =2 B \mu \sin (2 \theta) \varphi^{2} \\
& -(-1)^{k-1} 2(2 k-2) \lambda_{k}^{\prime} \mu \sin ((2 k-2) \theta) \varphi^{2 k}, \\
\frac{\partial^{2} \tilde{V}}{\partial \theta^{2}} & =4 B \mu \cos (2 \theta) \varphi^{2} \\
& -(-1)^{k-1} 2(2 k-2)^{2} \lambda_{k}^{\prime} \mu \cos ((2 k-2) \theta) \varphi^{2 k} .
\end{aligned}
$$

Considering principal values of $\theta, \tilde{V}(\varphi, \theta)$, which may posses a secondary minimum for $\theta \in\left\{0, \pm \frac{\pi}{2}\right\}$. To illustrate further, with $\theta=0$, we have

$$
\begin{aligned}
\frac{\partial V(\varphi)}{\partial \varphi}= & m_{0}^{2} \varphi+(-1)^{k-1} 4 k \lambda_{k}^{\prime} \mu \phi^{2 k-1} \\
& +4(2 k-1) \lambda_{k}^{\prime 2} \varphi^{4 k-3}, \\
\frac{\partial^{2} V(\varphi)}{\partial \varphi^{2}}= & m_{0}^{2}+(-1)^{k-1} 4 k(2 k-1) \lambda_{k}^{\prime} \mu \phi^{2(k-1)} \\
& +4(2 k-1)(4 k-3) \lambda_{k}^{\prime 2} \varphi^{4(k-1)},
\end{aligned}
$$

where, $V(\varphi)=\tilde{V}(\varphi, 0), m_{0}=m(\theta=0)$. The necessary conditions for the existence of the secondary local minimum, required by the reality of $\varphi$, are given by

$$
k^{2} \mu^{2} \geq m_{0}^{2}(2 k-1),
$$

and also the coefficient of $\varphi^{2 k-1}$ in Eq. (9) must be negative. At this minimum, we have

$$
\varphi=\varphi_{0} \sim\left(m_{0} M_{P}^{2 k-3}\right)^{1 / 2 k-2} \ll M_{P}
$$

The curvature of the potential along the radial direction is $m_{0}^{2}$, and the potential reduces to:

$$
V \sim m_{0}^{2} \varphi_{0}^{2} \sim m_{0}^{2}\left(m_{0} M_{P}^{2 k-3}\right)^{2 /(2 k-2)} .
$$

This is the situation when the flat direction can get trapped in the false minimum $\varphi_{0}$. If its potential energy,
$V$, dominates the total energy density of the universe, a period of inflation is obtained. However, one finds that $H_{\text {inf }} \sim m_{0} \varphi_{0} / M_{P} \sim m_{0}\left(m_{0} / M_{P}\right)^{1 /(2 k-2)} \ll m_{0}$. This implies that the potential is too steep at the false minimum and $\phi$ cannot climb over the barrier which separates the two minima just by the help of quantum fluctuations during inflation. The situation is essentially the same as in the old inflation scenario [14] with no graceful exit from inflation.

\section{INFLECTION POINT INFLATION}

This issue can be resolved if inflation occurs around an inflection point instead [4, [5, 15, 16]. The inflection point can be obtained if the following condition is satisfied,

$$
m_{0}^{2}=\frac{k^{2} \mu^{2}}{(2 k-1)}+\tilde{\lambda}^{2}
$$

where $\tilde{\lambda}$ is the tuning required to lift the potential ${ }^{3}$. If this relation holds good at the appropriate energy scale, an inflection point exists at $\varphi_{0}$, where,

$$
\begin{aligned}
\lambda^{2} & =\frac{2 k-1}{8(k-1)^{2}} \frac{\tilde{\lambda}^{2}}{\mu^{2} k^{2}}=\frac{\tilde{\lambda}^{2} m_{0}^{-2}}{8(k-1)^{2}}, \\
\varphi_{0} & =\left(\frac{k|\mu|\left|\lambda_{k}^{\prime}\right|^{-1}}{2(2 k-1)}\right)^{1 /(2 k-2)}\left(1-\lambda^{2}\right)+\mathcal{O}\left(\lambda^{4}\right), \\
& =\left(\frac{m_{0}\left|\lambda_{k}^{\prime}\right|^{-1}}{2 \sqrt{2 k-1}}\right)^{1 / 2 k-2}\left(1-\lambda^{2}\right)+\mathcal{O}\left(\lambda^{4}\right) .
\end{aligned}
$$

Around the inflection point $\varphi_{0}$, the potential for the inflaton may be expanded in a Taylor series,

$$
V(\varphi)=V_{0}+\alpha_{1}\left(\varphi-\varphi_{0}\right)+\frac{1}{3 !} \alpha_{3}\left(\varphi-\varphi_{0}\right)^{3}+\ldots .
$$

where, $V_{0}=V\left(\varphi_{0}\right), \alpha_{1}=\left.\frac{\partial V(\varphi)}{\partial \varphi}\right|_{\varphi=\varphi_{0}}$ and $\alpha_{3}=$ $\left.\frac{\partial^{3} V(\varphi)}{\partial \varphi^{3}}\right|_{\varphi=\varphi_{0}}$. Here we have assumed that,

$$
\begin{aligned}
& \left|\alpha_{1}\right| \gg\left|\frac{d^{n} V}{d \varphi^{n}}\right|_{\varphi=\varphi_{0}}\left|\varphi_{e}-\varphi_{0}\right|^{n-1}, \\
& \left|\alpha_{3}\right| \gg\left|\frac{d^{n} V}{d \varphi^{n}}\right|_{\varphi=\varphi_{0}}\left|\varphi_{e}-\varphi_{0}\right|^{n-3},
\end{aligned}
$$

where $n \geq 4$ and $\varphi_{e}$ denotes $\varphi$ at the end of slow-roll regime. In terms of the relevant parameters, we also

\footnotetext{
${ }^{3}$ When $k^{2} \mu^{2}=(2 k-1) m_{0}^{2}$, the potential accommodates a saddle point. In this paper we will follow the analysis of inflection point.
} 
have,

$$
\begin{aligned}
V_{0} & =\mu^{2} k \frac{(k-1)^{2}}{(2 k-1)^{2}}\left(\frac{k|\mu|\left|\lambda_{k}^{\prime}\right|^{-1}}{2(2 k-1)}\right)^{1 / k-1}+\mathcal{O}\left(\lambda^{2}\right) \\
& =\frac{(k-1)^{2} m_{0}^{2}}{k(2 k-1)} \varphi_{0}^{2}+\mathcal{O}\left(\lambda^{2}\right) \\
\alpha_{1} & =\tilde{\lambda}^{2}\left(\frac{k|\mu|\left|\lambda_{k}^{\prime}\right|^{-1}}{2(2 k-1)}\right)^{1 / 2 k-2}+\mathcal{O}\left(\lambda^{4}\right) \\
& =8(k-1)^{2} \lambda^{2} m_{0}^{2} \varphi_{0}+\mathcal{O}\left(\lambda^{4}\right) \\
\alpha_{3} & =8(k-1)^{2} \frac{m_{0}^{2}}{\varphi_{0}}+\mathcal{O}\left(\lambda^{2}\right)
\end{aligned}
$$

From now on we only keep the leading order terms in all the expressions. Note that the success of electroweak symmetry breaking requires the $\mu$-term and the soft breaking mass to be the same order of magnitude, $m_{0} \sim \mu \sim \mathcal{O}(100) \mathrm{GeV}$.

The slow-roll parameters, in the vicinity of the inflection point, are given by,

$$
\begin{aligned}
\epsilon(\varphi) & =\frac{1}{2}\left(\frac{V^{\prime}(\varphi)}{V(\varphi)}\right)^{2}=\frac{M_{P}^{2}}{2 V_{0}^{2}}\left(\alpha_{1}+\frac{\alpha_{3}}{2}\left(\varphi-\varphi_{0}\right)^{2}\right)^{2} \\
\eta(\varphi) & =M_{P}^{2} \frac{V^{\prime \prime}(\varphi)}{V(\varphi)}=M_{P}^{2} \frac{\alpha_{3}}{V_{0}}\left(\varphi-\varphi_{0}\right) \\
\xi(\varphi) & =M_{P}^{4} \frac{V^{\prime}(\varphi) V^{\prime \prime \prime}(\varphi)}{V(\varphi)^{2}} \\
& =M_{P}^{4} \frac{\alpha_{3}}{V_{0}^{2}}\left(\alpha_{1}+\frac{\alpha_{3}}{2}\left(\varphi-\varphi_{0}\right)^{2}\right) .
\end{aligned}
$$

The Hubble expansion rate during the slow-roll inflation is,

$$
H_{\text {inf }} \simeq \sqrt{\frac{V_{0}}{3 M_{P}^{2}}}=\frac{k-1}{\sqrt{3 k(2 k-1)}} \frac{m_{0} \varphi_{0}}{M_{P}} .
$$

For $\varphi_{0} \sim 10^{14} \mathrm{GeV}$, a typical VEV at the inflection point in our context ${ }^{4}$

$$
H_{\text {inf }} \sim \frac{m_{0} \phi_{0}}{M_{P}} \sim 10^{-1} \mathrm{GeV},
$$

Inflation ends when $|\eta| \simeq 1$. The interval suitable for inflation is given by,

$$
\frac{\left|\varphi_{0}-\varphi\right|}{\varphi_{0}} \sim\left(\frac{\varphi_{0}}{8 k(2 k-1) M_{P}}\right)^{2}
$$

The amplitude of density perturbation generated is given by [5, 15, 16],

$$
\begin{array}{r}
\delta_{H} \simeq \frac{1}{5 \pi} \sqrt{\frac{2}{3} 2 k(2 k-1)}(2 k-2)\left(\frac{m_{0} M_{P}}{\varphi_{0}^{2}}\right) \\
\frac{1}{\Delta^{2}} \sin ^{2}\left[\mathcal{N}_{\mathrm{COBE}} \sqrt{\Delta^{2}}\right]
\end{array}
$$

\footnotetext{
${ }^{4}$ The initial conditions for low scale inflation have been discussed in Refs. [17 19.
}

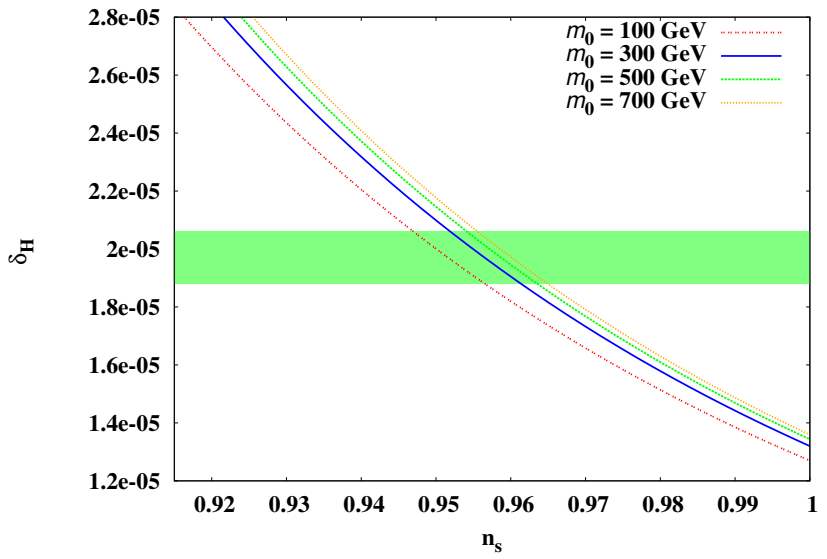

FIG. 1: $\delta_{H}$ and $n_{s}$ have been plotted for different values of $m_{0}$ and at the inflection point VEV, $\varphi_{0} \sim 3 \times 10^{14} \mathrm{GeV}$. We have used $k=2$ in the superpotential, we have taken $\lambda_{2}^{\prime} M_{P}=1.4 \times 10^{-8}$, see Eq. (6). The green band denotes $2 \sigma$ allowed band of $\delta_{H}$ [1]. Although the splitting between these curves are not so sensitive to the inflaton mass, varying $\lambda_{2}^{\prime}$ it is possible to span the complete range in the $n_{s}-\delta_{H}$ plane.

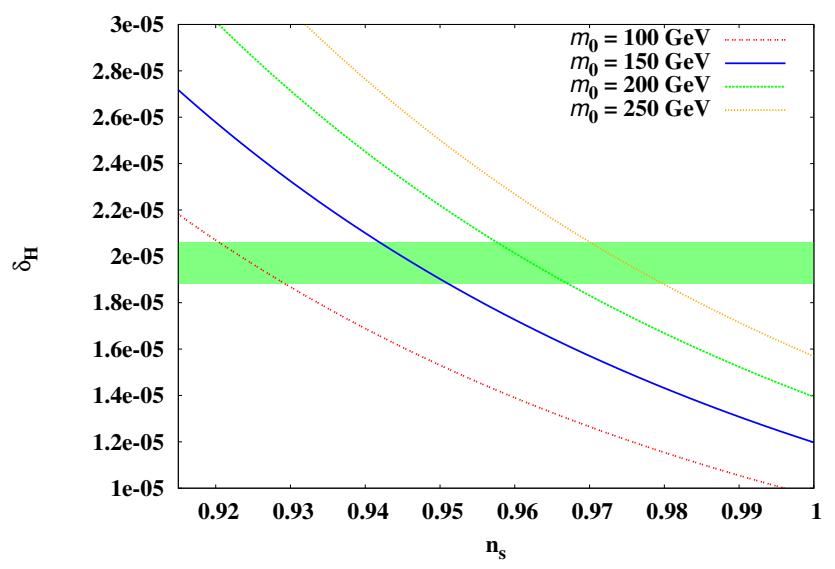

FIG. 2: $\delta_{H}$ and $n_{s}$ have been plotted for different values of $m_{0}$ and at the inflection point VEV, $\varphi_{0} \sim 3 \times 10^{14} \mathrm{GeV}$. We have used $k=3$ in the superpotential, we have taken $\lambda_{3}^{\prime} M_{P}^{3}=-0.71$, see Eq. (6). The green band denotes $2 \sigma$ allowed band of $\delta_{H}$ [1].

and the spectral index for the scalar perturbation is given by $[5,15,16]$,

$$
n_{s}=1-4 \sqrt{\Delta^{2}} \cot \left[\mathcal{N}_{\mathrm{COBE}} \sqrt{\Delta^{2}}\right],
$$

where,

$$
\Delta^{2}=32 k^{2}(2 k-1)^{2} \lambda^{2} \mathcal{N}_{\text {COBE }}^{2}\left(\frac{M_{P}}{\varphi_{0}}\right)^{4} .
$$

$\mathcal{N}_{\mathrm{COBE}}$ is the number of e-foldings between the time when the observationally relevant perturbations were generated till the end of inflation and is given by 


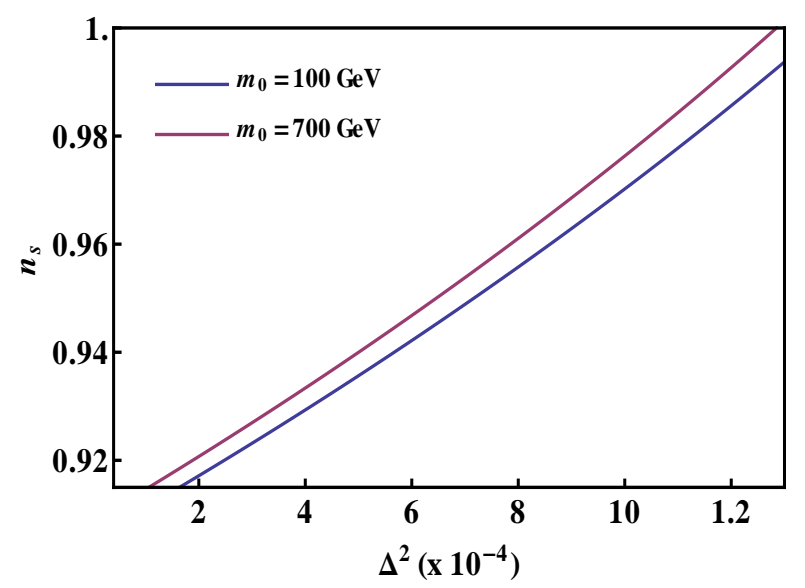

FIG. 3: $n_{s}$ has been plotted against vs $\Delta^{2}$ for different values of $m_{0}$ for $k=2$ case with $\lambda_{2}^{\prime} M_{P}=1.4 \times 10^{-8}$.

$\mathcal{N}_{\mathrm{COBE}} \simeq 66.9+(1 / 4) \ln \left(V\left(\varphi_{0}\right) / M_{P}^{4}\right) \sim 45$ 21], for either $k=2,3$, provided that the universe thermalizes within one-Hubble time after the end of inflation-see the discussion below.

So far the expressions hold good for any general $k$. Now let us consider two simple examples.

- $k=2$ case:

Let us consider the lowest order non-renormalizble term, with $k=2$. Using the general formulation we described, we plot $\delta_{H}$ vs $n_{s}$ in Fig. 11. In the context of low energy supersymmetry, with inflaton masses of about $100-1000 \mathrm{GeV}$, it is possible to obtain the required density perturbations and the spectral index, consistent with the WMAP data [1], as indicated by the figure, for the VEV around $\varphi_{0} \sim$ $10^{14} \mathrm{GeV}$.

However, the coefficient $\lambda_{2}$, which naturally may be expected to be of order $\mathcal{O}(1)$, needs to be of order $10^{-8}$, or to be more specific $\lambda_{2}^{\prime} M_{P} \sim 1.4 \times 10^{-8}$, see Eq. (6), instead. We note that there are other dimension 5 operators, even allowed by R-parity, which needs similar suppression to satisfy constraints from the proton deacy. These are $Q Q Q L$ and $\bar{U} \bar{U} \bar{D} \bar{E}$, see, for instance, [20].

- $k=3$ case:

On the other hand, assuming $\lambda_{2} \approx 0$ and considering only $k=3$ term may lead to a satisfactory result. It is possible to match the CMB observations for $\delta_{H}$ and $n_{s}$ for $\lambda_{3} \sim \mathcal{O}(1)$, or to be more specific $\lambda_{3}^{\prime} M_{P}^{3} \sim-0.71$, see Eq. (6). We demonstrate this in Fig. 2, where we assumed $\lambda_{2}=0{ }^{5}$.

\footnotetext{
${ }^{5}$ Strictly speaking we can keep both $\lambda_{3} \sim \mathcal{O}(1)$ and $\lambda_{2} \sim 10^{-8}$ at $\varphi_{0} \sim 10^{14} \mathrm{GeV}$.
}

Since inflation occurs at low scale, the tensor to scalar ratio remains small. From Fig. 3, we see that in order to obtain the spectral index, consistent with the recent data, we need

$$
\Delta^{2} \sim 10^{-3} \Longrightarrow \lambda \sim 10^{-11} .
$$

Although the figure assumes $k=2$, a similar plot may also be obtained with $k=3$. Further note that $\lambda$, see Eq. (15), remains of the same order for both cases, $k=2$ and $k=3^{6}$.

After inflation the coherent oscillations of the Higgses excite the MS(SM) degrees of freedom and reheat the universe.The thermalization time scale is much shorter than one Hubble time. The Higgses start coherent oscillations around their minimum denoted by $\varphi=0$, which is the point of enhanced gauge symmetry-where the entire SM gauge symmetry is restored. When ever the inflaton passes through the point of enhanced gauge symmetry, the zero mode of the inflaton condensate excites the massless modes of MSSM via non-perturbative phenomena 22, 23], and [5, 6], known as instant preheating [23, 24]. These are the massless modes which couple to the inflaton directly, for instance the degrees of freedom corresponding to $S U(3)_{C} \times S U(2)_{W} \times U(1)_{Y}$. At VEVs away from the minimum, the same modes become heavy and therefore it becomes kinematically unfavorable to excite them.

The actual process of excitation depends on how strongly the adiabaticity condition for the time dependent vacuum is violated for the inflaton zero mode 23 , 24]. Nevertheless, draining the inflaton energy is quite efficient, as shown in a particular MSSM flat direction inflaton $L L e$, where nearly $10 \%$ of the inflaton energy density gets transferred to the relativistic species. It takes near about 120 oscillations to reach the full chemical and kinetic equilibrium via processes requiring $2 \leftrightarrow 2$ and $2 \leftrightarrow 3$ interactions [6], given that one Hubble time corresponds to roughly 1000 oscillations. Note that the dynamical forms of the potentials for $L L e$ and $H_{1} H_{2}$ are very similar. Since $H_{1} H_{2}$ couples all the MS(SM) degrees of freedom, it is expected that the inflaton energy density would decay at a similar rate and thermalization time scale would be very similar to the case of $L L e$, which couples mainly to $S U(2)_{W} \times U(1)_{Y}$ degrees of freedom. In any case, due to the hierarchy in the scales, $m_{0} \sim 1 \mathrm{TeV}$, and $H_{\text {inf }} \sim 10^{-1} \mathrm{GeV}$, the thermalization time scale will be well short of 1000 inflaton oscillations, which marks the one Hubble time after inflation. For all practical purposes reheating and thermalization will be over instantly. The final reheat temperature will be given by:

$$
T_{\mathrm{rh}} \simeq\left(\frac{30}{\pi^{2} g_{*}}\right)^{1 / 4} \rho_{0}^{1 / 4} \simeq 2 \times 10^{8} \mathrm{GeV},
$$

\footnotetext{
${ }^{6}$ Although we have illustrated with $\theta=0$, a similar calculation may be performed with $\theta= \pm \pi / 2$, with appropriate choice of parameters.
} 
where we have used $g_{*}=228.75$ (all degrees of freedom in MSSM) and $\rho_{0}=(4 / 15) m_{0}^{2} \phi_{0}^{2}[6]$.

\section{FINE TUNING AND ELECTROWEAK SYMMETRY BREAKING}

Note that a fine tuning $(\lambda)$ of order $10^{-11}$ is required for inflation to occur, see Eqs. (15) 32). However, in our case the tuning involves only the MSSM parameters, although at a high scale. At one-loop level, these parameters evolve by the respective renormalization group equations (RGEs), as in the MSSM, see [26].

Ignoring the Yukawa couplings for the first two generations of fermions, the RGEs for $m_{1}, m_{2}, B, \mu$ are given by [26],

$$
\begin{aligned}
\frac{d \mu}{d \mathcal{E}}= & \frac{\mu}{16 \pi^{2}}\left(3 f_{t}^{2}+3 f_{b}^{2}+f_{\tau}^{2}-3 g_{2}^{2}-g_{Y}^{2}\right) \\
\frac{d B}{d \mathcal{E}}= & \frac{1}{8 \pi^{2}}\left(-3 f_{t}^{2} A^{t}-3 f_{b}^{2} A^{b}-f_{\tau}^{2} A^{\tau}+3 g_{2}^{2} M_{2}\right. \\
& \left.+g_{Y}^{2} M_{1}\right) \\
\frac{d m_{1}^{2}}{d \mathcal{E}}= & \frac{1}{8 \pi^{2}}\left(3 f_{b}^{2}\left(m_{1}^{2}+m_{\tilde{Q}_{3}}^{2}+m_{\tilde{b}_{R}}^{2}+\left|A^{b}\right|^{2}\right)\right. \\
& +f_{\tau}^{2}\left(m_{1}^{2}+m_{\tilde{l}_{3}}^{2}+m_{\tilde{\tau}_{R}}^{2}+\left|A^{\tau}\right|^{2}\right)-3 g_{2}^{2}\left|M_{2}\right|^{2} \\
& \left.-g_{Y}^{2}\left|M_{1}\right|^{2}-\frac{1}{2} g_{Y}^{2} S_{Y}\right) \\
\frac{d m_{2}^{2}}{d \mathcal{E}}= & \frac{1}{8 \pi^{2}}\left(3 f_{t}^{2}\left(m_{2}^{2}+m_{\tilde{Q}_{3}}^{2}+m_{\tilde{t}_{R}}^{2}+\left|A^{t}\right|^{2}\right)\right. \\
& \left.-3 g_{2}^{2}\left|M_{2}\right|^{2}-g_{Y}^{2}\left|M_{1}\right|^{2}+\frac{1}{2} g_{Y}^{2} S_{Y}\right)
\end{aligned}
$$

where $f_{i}$ s denote the Yukawa couplings for the i-th fermion species, $g_{2}$ and $g_{Y}$ denote the $S U(2)_{W}$ and $U(1)_{Y}$ gauge couplings respectively, $m_{i}$ s and $A^{i}$ s denote the soft supersymmetry breaking mass terms and the tri-linear terms respectively. $M_{i}$ s denote the soft supersymmetry breaking gaugino masses, and,

$$
S_{Y}=\frac{1}{2} \Sigma_{i} Y_{i} m_{i}^{2}
$$

where, the sum runs over all the scalar fields in MSSM, with masses $m_{i}$ and hypercharges $Y_{i}$. Also, all the other MSSM parameters involved in the above equations evolve simultaneously with $\mathcal{E}$ according to the respective RGEs.

Note that in our analysis, see Eq. (2), we have not considered any soft supersymmetry breaking term of the form $A \lambda_{k}^{\prime} \varphi^{2 k}$ in the Higgs sector. While this reduces the number of parameters involved, phenomenologically these terms are not forbidden. However, the origin of such a term may be determined by specific theories of supersymmetry breaking. Even if such a term is absent in the Higgs sector, similar terms involving Higgs fields and the sfermions can give rise to these terms at next to the leading order.

Considering a term of the form $A \lambda_{k}^{\prime} \varphi^{2 k}$ will lead to a straight forward modification of Eq. (14) removing any

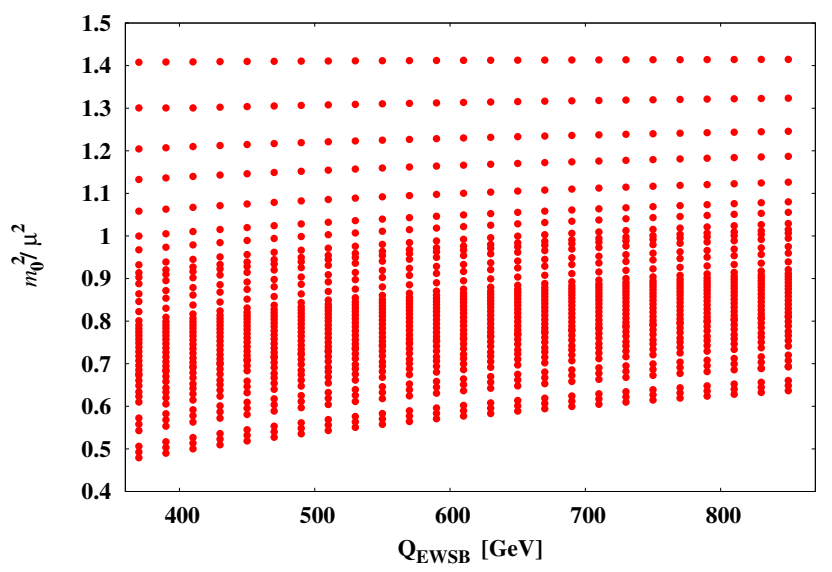

FIG. 4: A sample plot where the ratio $m_{0}^{2} / \mu^{2}$, see Eq. (14), for $k=2$, has been evaluated at the EWSB scale. The corresponding value at a high scale, $\varphi_{0} \sim 10^{14} \mathrm{GeV}$, is set to $4 / 3$, see Eq. (14), with an accuracy of $0.1 \%$. The RGE accuracy in SuSpect [27] is about $0.01 \%$.

tuning between $m_{0}$ and $\mu$. To illustrate, with $\theta=0$ and $\lambda_{k}^{\prime} A<0$, Eq. (14) will be modified to,

$$
m_{0}^{2}=\frac{k^{2}\left((-1)^{k-1} 2 \mu+A\right)^{2}}{4(2 k-1)}+\tilde{\lambda}^{2} .
$$

We demonstrate in Fig. (4), that various points in the parameter space, which give the tuning required at the scale of the VEV $\varphi_{0}$, do not satisfy any particular relation when evolved to the EWSB scale. The figure also shows that successful EWSB is possible over a large parameter space in spite of the tuning. We use the publicly available code SuSpect to evolve the RGEs [27], modifying it a little to cater our needs.

In Fig. (5) we evolve the ratio $m_{0}^{2} / \mu^{2}$ from a high scale, typical for $\varphi_{0}$, to the EWSB scale. The plot demonstrates that the ratio evolves with scale, and differs by about a part in 10 at the EWSB scale.

Thus the fine tuning between the parameters, as given by Eq. (14) may be satisfied dynamically. The relevant parameters, while assigning arbitrary $(\sim 1)$ values of $\lambda$ at a low scale, can still satisfy Eq. (14) at the scale of inflection point $\varphi_{0} \sim 10^{14} \mathrm{GeV}$.

\section{CONCLUSION}

To summarize, we have shown that a combination of MSSM Higgs fields can be the inflaton. A sufficient number of e-foldings and the right amount of density perturbations are obtained if, apart from the $\mu$ term, the $H_{1} H_{2}$ (D-)flat direction is lifted by $\left(H_{1} \cdot H_{2}\right)^{k}$ operator, for $k=2$, and $k=3$. However, for $k=2$, the coefficient $\lambda_{2}$ needs to be suppressed. The model predicts low scale inflation, non-detectable gravity waves, and a slight 


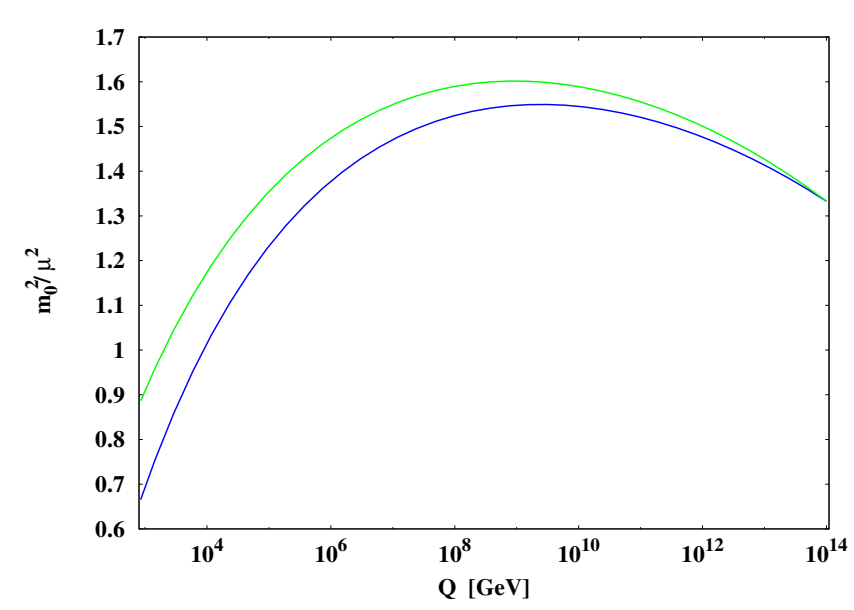

FIG. 5: The ratio $m_{0}^{2} / \mu^{2}$ for $k=2$, has been evolved from $10^{14} \mathrm{GeV}$ to the EWSB scale (chosen to be $850 \mathrm{GeV}$ ). The green line and the blue line correspond to $m_{0}=323.4 \mathrm{GeV}$ and $m_{0}=354 \mathrm{GeV}$ at $10^{14} \mathrm{GeV}$ respectively. The ratio at the high scale $\left(10^{14} \mathrm{GeV}\right)$ is set to $4 / 3$, see Eq. (14), with an accuracy of $0.1 \%$. The RGE accuracy in SuSpect [27] is about $0.01 \%$. departure from scale invariance. Further note that supergravity effects are negligible for $H_{\text {inf }} \ll m_{0}$ and therefore do not spoil the predictions [3, 5]. More importantly, there will be no moduli problem in our model. Since $H_{\text {inf }} \ll m_{0}$, all moduli will settle at their true minimum during inflation.

The salient feature of the present model is that the inflaton is now identified with the MSSM Higgs fields. Therefore its properties could be determined independently of cosmology by particle physics experiments. In particular the condition in Eq. (14) for having a flat potential amounts to a relationship among the parameters of the Higgs sector which can be determined at the LHC. Further work is required to establish these connections.

Acknowledgments- We wish to thank Rouzbeh Allahverdi, Manuel Drees and Jean-Loic Kneur for helpful discussions.
[1] E. Komatsu et al. [WMAP Collaboration], Astrophys. J. Suppl. 192 (2011) 18

[2] A. Mazumdar, J. Rocher, Phys. Rept. 497 (2011) 85215.

[3] R. Allahverdi, K. Enqvist, J. Garcia-Bellido and A. Mazumdar, Phys. Rev. Lett. 97, 191304 (2006)

[4] R. Allahverdi, A. Kusenko and A. Mazumdar, JCAP 0707, 018 (2007)

[5] R. Allahverdi, K. Enqvist, J. Garcia-Bellido, A. Jokinen and A. Mazumdar, JCAP 0706, 019 (2007)

[6] R. Allahverdi, A. Ferrantelli, J. Garcia-Bellido and A. Mazumdar, arXiv:1103.2123 [hep-ph].

[7] R. Allahverdi, B. Dutta and A. Mazumdar, Phys. Rev. D 75 (2007) 075018 arXiv:hep-ph/0702112. R. Allahverdi, B. Dutta and A. Mazumdar, Phys. Rev. Lett. 99 (2007) 261301

[8] R. Allahverdi, B. Dutta and Y. Santoso, Phys. Rev. D 82, 035012 (2010).

[9] M. Cicoli, A. Mazumdar, JCAP 1009, 025 (2010). M. Cicoli, A. Mazumdar, arXiv:1010.0941 [hep-th]].

[10] M. Pospelov, A. Ritz, Annals Phys.318: 119-169, 2005.

[11] M. Dine, L. Randall and S. D. Thomas, Nucl. Phys. B 458 (1996) 291 arXiv:hep-ph/9507453. T. Gherghetta, C. F. Kolda and S. P. Martin, Nucl. Phys. B 468 (1996) 37 arXiv:hep-ph/9510370.

[12] For reviews, see: K. Enqvist and A. Mazumdar, Phys. Rept. 380, 99 (2003). M. Dine and A. Kusenko, Rev. Mod. Phys. 76, 1 (2004).

[13] K. Enqvist, S. Kasuya and A. Mazumdar, Phys. Rev. Lett. 93 (2004) 061301 arXiv:hep-ph/0311224.

[14] A. H. Guth, Phys. Rev. D 23, 347 (1981).

[15] J. C. B. Sanchez, K. Dimopoulos and D. H. Lyth, JCAP 0701, 015 (2007).

[16] K. Enqvist, A. Mazumdar and P. Stephens, JCAP 1006
(2010) 020 arXiv:1004.3724 [hep-ph]]. S. Hotchkiss, A. Mazumdar and S. Nadathur, arXiv:1101.6046 [astroph.CO].

[17] R. Allahverdi, A. R. Frey and A. Mazumdar, Phys. Rev. D 76, 026001 (2007) arXiv:hep-th/0701233.

[18] R. Allahverdi, B. Dutta and A. Mazumdar, Phys. Rev. D 78 (2008) 063507 arXiv:0806.4557 [hep-ph]].

[19] K. Kamada, J. Yokoyama, Prog. Theor. Phys. 122 (2010) 969

[20] R. Harnik, D. Larson, H. , M. Thormeier, Nucl. Phys. B 706 (2005) 372 [arXiv: 0404260 [hep-ph]].

[21] A. R. Liddle and S. M. Leach, Phys. Rev. D 68, 103503 (2003) arXiv:astro-ph/0305263. C. P. Burgess, R. Easther, A. Mazumdar, D. F. Mota and T. Multamaki, JHEP 0505, 067 (2005).

[22] J. H. Traschen and R. H. Brandenberger, Phys. Rev. D 42 (1990) 2491. Y. Shtanov, J. H. Traschen and R. H. Brandenberger, Phys. Rev. D 51 (1995) 5438 arXiv:hep-ph/9407247.

[23] L. Kofman, A. D. Linde and A. A. Starobinsky, Phys. Rev. Lett. 73 (1994) 3195 arXiv:hep-th/9405187. L. Kofman, A. D. Linde and A. A. Starobinsky, Phys. Rev. D 56 (1997) 3258 arXiv:hep-ph/9704452.

[24] G. N. Felder, L. Kofman and A. D. Linde, Phys. Rev. D 59 (1999) 123523 arXiv:hep-ph/9812289.

[25] S. Weinberg, arXiv:hep-th/9803099

[26] M. Drees, R. Godbole and P. Roy, "Theory and phenomenology of sparticles: An account of fourdimensional $\mathrm{N}=1$ supersymmetry in high energy physics," SPIRES entry Hackensack, USA: World Scientific (2004) $555 p$

[27] A. Djouadi, J. L. Kneur and G. Moultaka, Comput. Phys. Commun. 176 (2007) 426 arXiv:hep-ph/0211331. 